\title{
Redes de Explicitación del Conocimiento y su Relación con la Productividad en Pymes
}

\author{
Giselle Pinochet ${ }^{1 \star}$
}

Resumen: En este estudio se presenta un modelo para evaluar la correlación entre la explicitación del conocimiento y la productividad de las empresas. El modelo se basa en ciencia de redes, y en un algoritmo de agrupamiento difuso, y se aplica a una muestra de 40 pymes colombianas. Los resultados muestran que las medidas de red seleccionadas como indicadores del modelo tienen alta y significativa correlación con la productividad, y permiten agrupar a las empresas en cuatro categorías de desempeño. Se analizan las implicaciones de los hallazgos, como aporte para superar el reto de medir procesos afines a la gestión del conocimiento y se proponen futuras líneas de investigación a partir de las limitaciones del estudio.

Palabras clave: explicitación del conocimiento, ciencia de redes, agrupamiento difuso, pymes.

Abstract: This study presents a model is presented to evaluate the correlation between the clarification of knowledge and the productivity of companies. The model is based on network science, and on a fuzzy clustering algorithm, and is applied to a sample of 40 Colombian SMEs. The results show that the network measures selected as indicators of the model have a high and significant correlation with productivity and allow companies to be clustered into four performance categories. The implications of the findings are analyzed, as a contribution to overcome the challenge of measuring processes related to knowledge management and future lines of research are proposed based on the limitations of the study.

Keywords: knowledge externalization, network science, fuzzy clustering, SME's

Submitted: March 18 ${ }^{\text {th }}, 2021 /$ Approved: April 24 $4^{\text {th }}, 2021$

\section{Introducción}

La Gestión del Conocimiento (GC) reviste una gran importancia para las empresas, porque éstas deben competir en mercados globales que demandan un uso extensivo del conocimiento (Dahou, Hacini, Burgoyne. 2019). De esa forma, la disciplina administrativa de la GC se convierte en el medio a través del cual se genera y aprovecha el conocimiento que las empresas poseen. Se ha propuesto que los esfuerzos en la GC deben orientarse a la creación de conocimiento, siendo este proceso en gran medida responsable de los mejores resultados obtenidos por las organizaciones (Deni, et.al., 2020; Ferreira, Mueller \& Papa, 2018; Kao \& Wu, 2016; Nonaka \& Takeuchi, 1995), especialmente en la productividad (Casaburi et al, 2016; Nonaka, Toyama \& Nagata, 2000; Uwasomba et.al, 2016).

Por lo tanto, uno de los principales propósitos de la GC es la creación del conocimiento. Una las formas más eficientes hacerlo es a través del proceso denominado transformación de conocimiento, en la cual los trabajadores toman el conocimiento ya existente, y lo integran para dar lugar a nuevo conocimiento (Bashir\& Farooq, 2019). La transformación del conocimiento es un proceso social porque depende de la interacción entre personas. Involucra el diálogo e intercambio de experiencias, y en consecuencia su eficacia depende de la calidad de las relaciones entre los trabajadores (Nonaka, Toyama \& Nagata, 2000; Rai and Prakash, 2012; Obeidat, 2019).

Por dicha razón es indispensable que la empresa asuma la responsabilidad de fomentar las interacciones a través del trabajo en equipo y la cooperación en la resolución de problemas (Kao and Wu, 2016; Takeuchi, 2001). Esto da lugar a la construcción conjunta de nuevo conocimiento y nuevas capacidades que mejoran las prácticas, procesos, formas de abordar las situaciones y superar nuevos retos. Pese a que los cuatro procesos de transformación de conocimiento son necesarias para las organizaciones, la explicitación de conocimiento (EC) puede aportar más valor ya permite generar un activo disponible permanentemente. (Kyakulumbye, Pather, Jantjies, 2019; Smith, 2001). Adicionalmente, genera conocimiento libre de restricciones de espacio y tiempo, con mínimo riesgo de pérdida. Una ventaja adicional del conocimiento creado en la EC es que se pueden identificar necesidades de conocimiento, lo que impulsa nuevos procesos de creación.

No obstante los beneficios el fomento de la EC, persiste el desafío de evaluar cuantitativamente su relación con el desempeño general de la empresa. La naturaleza intangible de este proceso conocimiento hace que medirlo, y medir su impacto en la empresa, siga siendo un reto que aún no se ha resulto por completo (Chatterjee, Pereira, \& Sarkar, 2018). Y persiste en el estudio sobre la gestión del conocimiento el debate sobre las implicaciones prácticas que tiene esta actividad sobre el desempeño real de las empresas (Alkhazali, Aldabbagh, \& AbuRumman, 2019; Deni, et.al., 2020).

Por su parte, las pequeñas y medianas empresas (Pymes), especialmente en Latinoamérica, enfrentan una dificultad adicional a la medición del proceso de EC. La falta de talento humano especializado en el tema, así como escasez de competencias organizacionales tienden

Facultad de Ciencias Económicas y Administrativas, Universidad Antonio Nariño, Bogotá, Colombia

*Autor de correspondencia: gpinochet@uan.edu.co 
a ser los principales obstáculos a la hora de llevar a cabo la GC en Pymes (Dini, Stumo \& Italiana, 2011; Frohmann, Mulder \& Olmos, 2018). Es por ello que este trabajo se propone como objetivo diseñar un modelo que permita evaluar cuantitativamente la relación entre el proceso de EC y la productividad en pymes.

\section{Revisión de la literatura}

En las organizaciones existen dos tipos de conocimiento, el tácito y el explícito. El conocimiento tácito se caracteriza por ser subjetivo y difícil de gestionar; en contraste, el conocimiento explícito está documentado y puede y gestionarse (Kyakulumbye, Pather, Jantjies, 2019; Smith, 2001). A partir de estas dos formas de conocimiento, tácito y explicito, se establecen cuatro procesos de transformación de conocimiento: socialización (de tácito a tácito), explicitación (de tácito a explícito), combinación (de explícito a explícito), e interiorización (de explícito a tácito). Los trabajos sobre transformación del conocimiento indican que este proceso tiene tres características fundamentales. Primero, no son las empresas las que crean conocimiento, sino que se valen de sus trabajadores para hacerlo (Nonaka \& Takeuchi, 1995). Segundo, es un proceso esencialmente social porque depende de la interacción entre los trabajadores de la organización que intercambian experiencias para crear nuevo conocimiento (Rai \& Prakash, 2012; Obeidat, 2019). Tercero, es un proceso dinámico, cambiante y continuo (Hemmecke \& Stary, 2004; Nonaka, Toyama \& Nagata, 2000).
Existen unas condiciones llamadas facilitadores de los procesos de transformación del conocimiento en las empresas (Nonaka \& Takeuchi, 1995). La primera de ellas es la necesidad de interacción entre los trabajadores para que dicho proceso tenga lugar (Nonaka, Von Krogh, \& Voelpel, 2006). Segundo, el acceso y la equidad en el acceso al conocimiento creado a partir de la TC de parte de todos los trabajadores de la empresa es necesario para que fomentar el ciclo de creación (Nonaka, Toyama \& Nagata, 2000). Finalmente, que la empresa garantice las condiciones para la TC a través de un ambiente favorable que estimule la creatividad y aliente a los trabajadores a abordar nuevas formas de resolver los problemas (Alvarenga Neto \& Choo, 2011; Kyakulumbye, Pather, Jantjies, 2019).

$\mathrm{Al}$ ser la EC un proceso derivado de la interacción entre los trabajadores resulta conveniente la aplicación de una herramienta como la ciencia de redes, la cual permite la medición y evaluación de procesos relacionales (Barabási, 2016). Modelar un sistema en forma de red, significa simplificar su realidad en términos de nodos y vínculos, las unidades fundamentales de una red. Para modelar y cuantificar las propiedades que surgen de la complejidad de la interacción, se emplean las medidas de red, indicadores cuantitativos del comportamiento de una red (Boccaletti, Latora, Moreno, Chavez, \& Hwang, 2006). Un conjunto de las medidas más comúnmente utilizadas se encuentra en la Tabla 1, clasificadas en dos categorías: medidas de red propiamente dichas, que evalúan características generales de todo el sistema; y medidas de nodo que evalúan características individuales.

Tabla 1: Definición de algunas de las principales medidas de red.

\begin{tabular}{|c|c|c|}
\hline Tipo de medida & Medida & Definición \\
\hline \multirow{3}{*}{ De red } & Diámetro & Es el máximo valor en el conjunto de todas las distancias de la red. \\
\hline & Distancia & Es el camino con el menor número de vínculos entre dos nodos. \\
\hline & Coeficiente de agrupamiento & $\begin{array}{l}\text { El coeficiente de agrupamiento local mide la densidad local de enlaces entre los vecinos } \\
\text { de un nodo. El coeficiente de agrupamiento es el promedio de los coeficientes de agrupa- } \\
\text { miento local de la red }\end{array}$ \\
\hline De nodo & Centralidad de cercanía & Es la inverso del promedio de las distancias entre un nodo y todos los demás nodos. \\
\hline
\end{tabular}

Fuente: elaboración propia con base en Barabási, 2016 y Boccaletti, Latora, Moreno, Chavez, \& Hwang, 2006.

Diferentes estudios han aplicado la ciencia de redes para evaluar procesos relacionados con el conocimiento organizacional. Por ejemplo, se han evaluado procesos como la capacidad de creación de conocimiento intra organizacional, transferencia de conocimiento, aprendizaje organizacional y capacidades de innovación (Hahn, Lee y Lee, 2015; Jiang, Zhang, Cheng, Xu, De Pablos, \& Wang, 2019; Phelps, Heidl, \& Wadhwa, 2012; Shin \& Kook, 2014; Sutanto, Tan, Battistini, \& Phang, 2011; Yun \& Lee, 2013). Para evaluar tales procesos, se han empleado medidas como la densidad, centralidad de grado, centralidad de grado y peso del vínculo. Los resultados de estos trabajos permiten concluir que algunas medidas de red son más favorables para cuantificar cierto tipo de procesos de conocimiento, pero no existen características universalmente útiles para todo tipo de procesos.

\section{Metodología}

La construcción y validación del modelo de red para medir la relación entre EC de conocimiento y productividad se llevó a cabo en tres etapas que se describen a continuación.

Planteamiento del modelo de evaluación. El modelo se construye para evaluar la correlación entre dos variables: la productividad y los procesos de EC. La productividad se mide con el indicador de productividad definido por la ecuación (1):

$$
p=I / T
$$


donde $p$ es el índice de productividad, $I$ los ingresos brutos en millones de pesos colombianos y $T$ corresponde al número de puestos de trabajo de la empresa.

Mientras que la EC se evalúa a partir de cuatro medidas de red, descritas en términos de nodos y vínculos. Los nodos de la red de EC representan los trabajadores de una empresa y los vínculos representan una relación entre los trabajadores que surge si participan juntos en al menos un proceso de EC. Esto es, que dos trabajadores participen juntos en al menos la creación de un registro escrito -como una base de datos o un protocolo- y puesta a disposición de la empresa. No se consideran, por lo tanto, documentos como notas o archivos personales que sirven exclusivamente a un trabajador. A continuación se describen las medidas de red empleadas como indicadores del modelo.

Diámetro. Formalmente está definida por la ecuación (2):

$$
D=d_{i j} \max
$$

donde $d_{i j}$ es el camino más corto entre los nodos $i$ y $j$. Los valores que puede tomar esta medida en una red de una red están en el rango $1<D<n-1$ donde $n$ es el número de nodos.

El diámetro se ve afectado por tanto por el número de vínculos como por la posición éstos ocupan dentro de la red. Al ser una medida que revela características de la distribución de los vínculos, permite cuantificar la distribución de los procesos de EC entre los trabajadores.

La Figura 1 ilustra la relevancia de esta distribución, a través de una red de cuatro nodos. En $a$ se muestra la red en un estado inicial con diámetro inicial es igual a 2 . En el bloque $b$ se muestra la misma red a la que se ha añadido un nuevo vínculo y se obtiene un diámetro de 3 y en el bloque $c$ se muestra la red inicial a la que el nuevo vínculo se añadió en una posición diferente a la anterior y se obtiene un diámetro de 2 nuevamente.

Se propone esta medida como variable del modelo porque permite evaluar el acceso de los trabajadores al conocimiento creado por otros. En redes con un alto valor en el diámetro, superior a 4, los nodos tienden a tener dificultad para conectarse entre ellos.

Figura 1: Ejemplo de red con un mismo estado inicial y dos posibles estados finales en donde la posición del vínculo varía el valor de la medida diámetro.

\begin{tabular}{|l|l|l|}
\hline & Red original & Red alternativa 1 \\
\hline Diámetro $=2$ & Diámetro=3 & Diámetro=2 \\
\hline
\end{tabular}

Fuente: elaboración propia.

Densidad. La densidad $\delta$ de una red está definida formalmente por la ecuación (3):

$$
\delta=\frac{2 l}{n(n-1)}(3)
$$

donde $n$ es el número de nodos y $l$ el número de vínculos de la red. Los valores que puede tomar esta medida están en el rango $1<\delta<1$. Esta medida sólo se ve afectada por el número de vínculos, no por su distribución en la red, ya que la posición en la red en que éstos se añadan no varía su resultado. Se propone esta medida como variable del modelo porque permite evaluar la cantidad de interacción entre los trabajadores en los procesos de EC.
Coeficiente de agrupamiento. Esta medida está definida formalmente por la ecuación (4):

$$
\bar{C}=\frac{1}{n} \sum_{i \in N} C_{i}
$$

donde $C_{i}$ es el coeficiente de agrupamiento del nodo $i$. Los valores que puede tomar esta medida están en el rango $1<\bar{C}<1$.

El agrupamiento local mide la densidad local entre los vecinos de un nodo y el coeficiente de agrupamiento es el promedio de los agrupamientos locales de toda la red (Barabási, 2016). Esta medida se ve afectada por el número de vínculos y su localización dentro de la red. 
En la Figura 2 se puede observar que un nuevo vínculo puede incrementar o disminuir el valor de la medida, de manera similar a lo que ocurre con el diámetro. En el bloque $a$, se muestra una red de cinco nodos y seis vínculos, cuyo coeficiente de agrupamiento es 0,5 . En el bloque $b$ muestra la misma red a la que se ha añadido un nuevo vínculo cuyo efecto es disminuir el coeficiente de agrupamiento a 0,3. En el bloque $c$ se muestra la red inicial a la que el nuevo vínculo se añadió en una posición diferente a la anterior con lo cual el coeficiente de agrupamiento aumenta en relación con la posición inicial y el valor de esta medida es de 0,63 .

Se propone esta medida como variable del modelo porque permite evaluar la cercanía entre los trabajadores en los procesos de EC.

Figura 2: Ejemplo de red con un mismo estado inicial y dos posibles estados finales en donde la posición del vínculo varía el valor de la medida coeficiente de agrupamiento.

\begin{tabular}{|l|l|l|}
\hline \multicolumn{2}{|c|}{ Red original } & Red alternativa 1 \\
\hline Coeficiente de agrupamiento $=0,5$ & Coeficiente de agrupamiento $=0,3$ & Coeficiente de agrupamiento $=0,63$ \\
\hline
\end{tabular}

Fuente: elaboración propia.

Desviación estándar de centralidad de cercanía. Está definida por la ecuación (5):

$$
\operatorname{Dscc}(i)=\sigma\left(\Sigma_{j}\left[d_{i j}\right]^{-1}\right)
$$

donde $i$ es el nodo focal, que es al que se calcula la centralidad, $j$ es el nodo hasta el cual se calcula la distancia a partir del nodo focal y $d_{i j}$, al igual que en la definición del diámetro, es el camino más corto entre los nodos $i$ y $j$ (Boccaletti et.al, 2006). La Dscc es una medida que caracteriza toda la red, puesto que se calcula la desviación estándar a la centralidad de cercanía de todos los nodos, lo cual es representado por $\sigma$. En este sentido, es una medida de la desigualdad: altos valores de desviación estándar implican que algunos nodos son muy centrales, y otros muy periféricos. Se propone esta medida como variable del modelo porque permite evaluar el nivel de dificultad que tienen los trabajadores para acceder a los procesos de EC en los que no participaron.

Levantamiento de información y descripción de la muestra. Para aplicar el modelo a las empresas se requiere información sobre su productividad y procesos de EC. Con el propósito de obtener los datos requeridos se aplicó un formulario virtual para el levantamiento de información, como se muestra en la Tabla 2. Con la información sobre ingresos y número de puestos de trabajo se calcula la productividad aplicando la ecuación (1). Y con la información sobre el listado de puestos de trabajo, documentos y puestos de trabajo involucrados en documento se construye una red por empresa y se calculan las respectivas medidas.

Tabla 2: Formulario de levantamiento de información aplicado a las empresas de ambas muestras.

\begin{tabular}{l|l}
\hline Nombre de la empresa & \\
\hline Fecha & \\
\hline Ingresos por ventas del último año & \\
\hline Listado de puestos de trabajo & \\
\hline
\end{tabular}

\begin{tabular}{l|l|l}
\hline & Nombre del documento & Puestos de trabajo participantes \\
\hline Documento 1 & & \\
\hline Documento 2 & & \\
\hline
\end{tabular}

Fuente: elaboración propia. 
Se obtuvo información de una muestra de 40 Pymes colombianas de diferentes actividades económicas, siendo criterios de inclusión el tamaño y nacionalidad de la empresa. Para el levantamiento de información se envió un formulario electrónico que recrea las características presentadas en la Tabla 2, junto con una declaración de privacidad de la información por lo que el nombre de la organización se empleó únicamente para identificar a las empresas al interior del estudio. La información fue recopilada en el período comprendido entre julio y diciembre de 2019.

La Figura 3 muestra la distribución de las empresas de acuerdo con su actividad económica, y la Tabla 3 presenta el número de puestos de trabajo y la productividad, calculada con la ecuación (1) y con base en la información recolectada de las empresas.

Figura 3: Distribución de las 40 empresas de la muestra de construcción de acuerdo con su actividad económica.

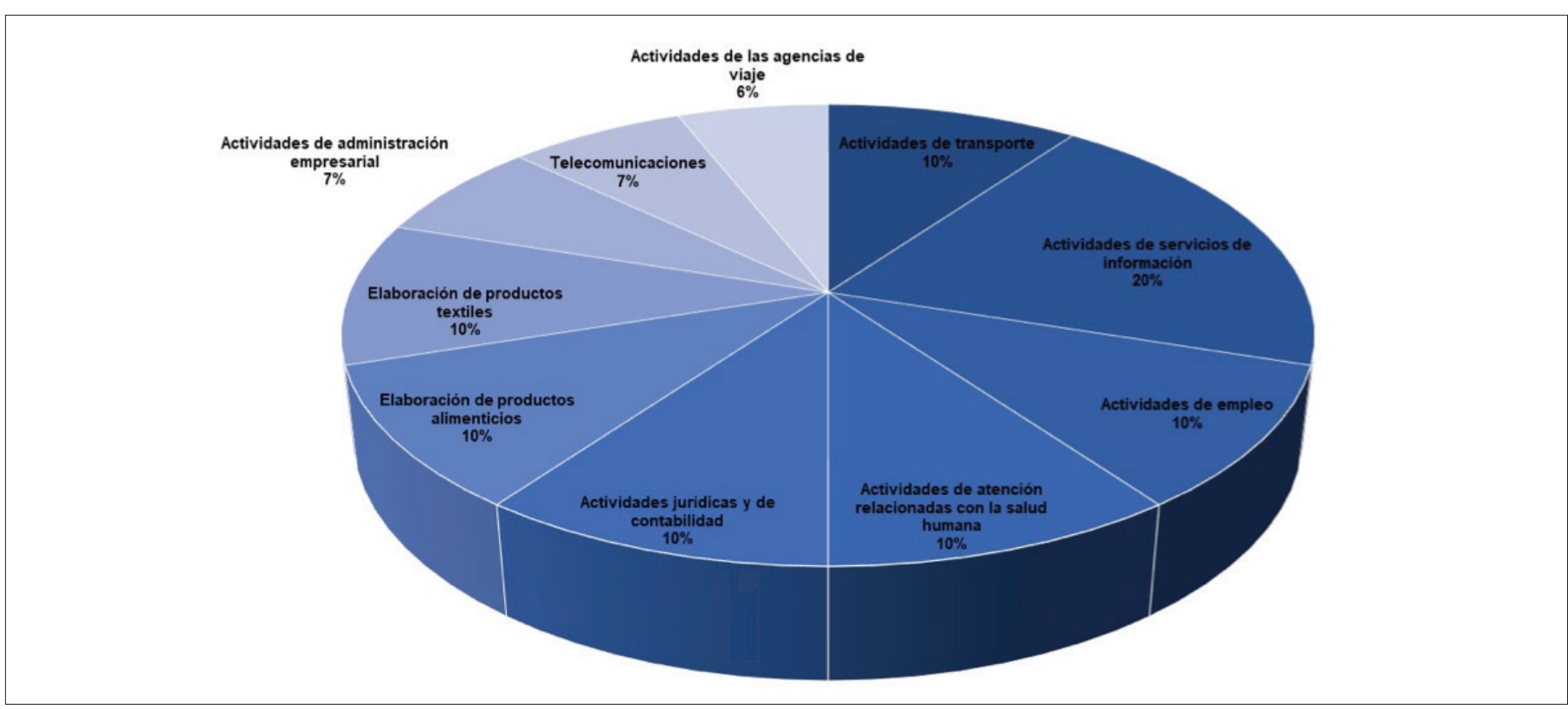

Fuente: elaboración propia.

Tabla 3: Información de las empresas de la muestra

\begin{tabular}{lll}
\hline Empresa & Productividad & $\boldsymbol{N}^{\circ}$ puestos de trabajo \\
\hline 1 & 25.71 & 23 \\
\hline 2 & 26.83 & 22 \\
\hline 3 & 31.51 & 19 \\
\hline 4 & 31.30 & 20 \\
\hline 6 & 54.85 & 17 \\
\hline 7 & 54.59 & 19 \\
\hline 8 & 54.24 & 18 \\
\hline 9 & 30.23 & 41 \\
\hline 10 & 43.27 & 32 \\
\hline 12 & 45.73 & 55 \\
\hline 13 & 70.06 & 14 \\
\hline 14 & 53.65 & 33 \\
\hline 15 & 66.16 & 25 \\
\hline 16 & 56.34 & 34 \\
\hline 17 & 44.73 & 61 \\
\hline 18 & 65.16 & 21 \\
\hline 19 & 57.15 & 20 \\
\hline 20 & 55.14 & 17 \\
\hline
\end{tabular}

\begin{tabular}{lll}
\hline Empresa & Productividad & $\boldsymbol{N}^{\circ}$ puestos de trabajo \\
\hline 21 & 65.66 & 15 \\
\hline 22 & 55.65 & 19 \\
\hline 23 & 65.96 & 20 \\
\hline 24 & 64.86 & 22 \\
\hline 25 & 56.06 & 18 \\
\hline 26 & 65.96 & 21 \\
\hline 27 & 45.69 & 16 \\
\hline 28 & 45.69 & 16 \\
\hline 29 & 70.08 & 29 \\
\hline 30 & 56.05 & 19 \\
\hline 32 & 32.75 & 19 \\
\hline 33 & 56.11 & 17 \\
\hline 34 & 55.52 & 18 \\
\hline 35 & 67.26 & 21 \\
\hline 36 & 64.84 & 29 \\
\hline 37 & 43.39 & 63 \\
\hline 38 & 56.05 & 20 \\
\hline 39 & 63.74 & 24 \\
\hline 40 & 64.54 & 14 \\
\hline
\end{tabular}

Fuente: elaboración propia. 
Aplicación del modelo. En esta fase se evaluaron dos capacidades del modelo de red: la de establecer una correlación con la productividad, y la de discriminar las empresas de acuerdo con su nivel de productividad. Para el primer caso se aplicó la prueba Shapiro-Wilk para conocer si las variables tienen distribución normal, y como se presentará en la sección de resultados, ni la productividad ni las cuatro variables mostraron distribución normal. Por esta razón se seleccionó el coeficiente de correlación de Spearman, aplicable a variables cuantitativas sin distribución normal. El coeficiente de Spearman puede tomar valores entre - 1 y 1 , donde los valores negativos indican una correlación inversa entre las dos variables y los valores positivos una correlación directa. La fortaleza de la correlación está determinada por el valor absoluto del coeficiente: valores entre 0.0 y 0.34 se consideran de baja $\theta$ correlación, entre 0.35 y 0.65 se consideran de media, y entre 0.66 y 1 se consideran valores de alta correlación.

Para evaluar el criterio de discriminación de empresas se aplicó un algoritmo de agrupamiento difuso. Esto permite agrupar datos cuando las fronteras de los conjuntos no están claramente diferenciadas, como es el caso del presente estudio ya que no se establecen criterios para clasificar las empresas. El algoritmo empleado es fuzzy c-means (Bezdek, Ehrlich, \& Full, 1984) en el cual cada elemento pertenece a todos los conjuntos y la pertenencia a cada conjunto de cada elemento se mide por un coeficiente de pertenencia que toma valores entre 0 y 1 , donde 0 indica la no pertenencia y el 1 indica la pertenencia total. La suma de los coeficientes de pertenencia de un elemento a todos los conjuntos es de 1 , indicando que cada elemento debe pertenecer completamente a los conjuntos posibles, independientemente de su pertenencia parcial a cada uno. La pertenencia se calcula con base en la distancia euclidiana de cada elemento al centroide del conjunto, un elemento ficticio localizado donde la suma de las distancias de todos los elementos del conjunto al centroide es mínima. El centroide se emplea, además, como valor estándar de cada conjunto. Se fijó en 0,8 el coeficiente de pertenencia requerido para afirmar que una empresa pertenece a un conjunto determinado, pero no se estableció previamente el número de conjuntos. Se buscó que cada conjunto represente adecuadamente a los elementos a través del coeficiente de pertenencia, empezando por tres conjuntos. Si los elementos no presentan el coeficiente de pertenencia mínimo se aumenta el número de conjuntos para lograr una mejor representatividad.

Se aplicó el algoritmo para agrupar a las empresas con base en productividad y con base en los valores de las medidas de red. La capacidad de discriminación del modelo depende de que los conjuntos de empresas creados con base en productividad coincidan con los conjuntos de empresas creados con base en las medidas de red. Para este propósito se emplea la ecuación (6), que mide el coeficiente de coincidencia entre los conjuntos:

$$
C_{c}=\frac{N_{c, i}}{N_{t, i}}(6)
$$

donde $C_{c} N_{c, i}$ es el número de empresas coincidentes entre los conjuntos según productividad y según medidas de red, y $N_{t, i}$ es el número total de empresas.

\section{Resultados}

La Tabla 4 presenta los resultados obtenidos de la aplicación del test de Shapiro Wilk, que indican una distribución no normal de las medidas de red y del indicador de productividad.

Tabla 4. Resultados del test de normalidad Shapiro Wilk aplicado a la productividad y las cuatro medidas de red para ambas muestras. La significancia inferior a 0,05 indica que la variable no tiene distribución normal.

\begin{tabular}{|c|c|c|}
\hline Muestra & Variable evaluada & Significancia de la prueba \\
\hline \multirow{3}{*}{ Construcción } & Productividad & 0,034 \\
\hline & Densidad & 0,011 \\
\hline & Desviación estándar de centralidad de cercanía & 0,028 \\
\hline
\end{tabular}

Fuente: elaboración propia.

Dada la no normalidad de las variables, se aplicó el coeficiente de correlación de Spearman entre las variables de EC y la productividad. Además de una alta correlación, las medidas mostraron una alta significancia, lo que significa que la correlación obtenida no es producto del azar. Los resultados de la correlación de se muestran en la Figura 4. 
Figura 4: Coeficiente de correlación de Spearman entre cada medida y la productividad.

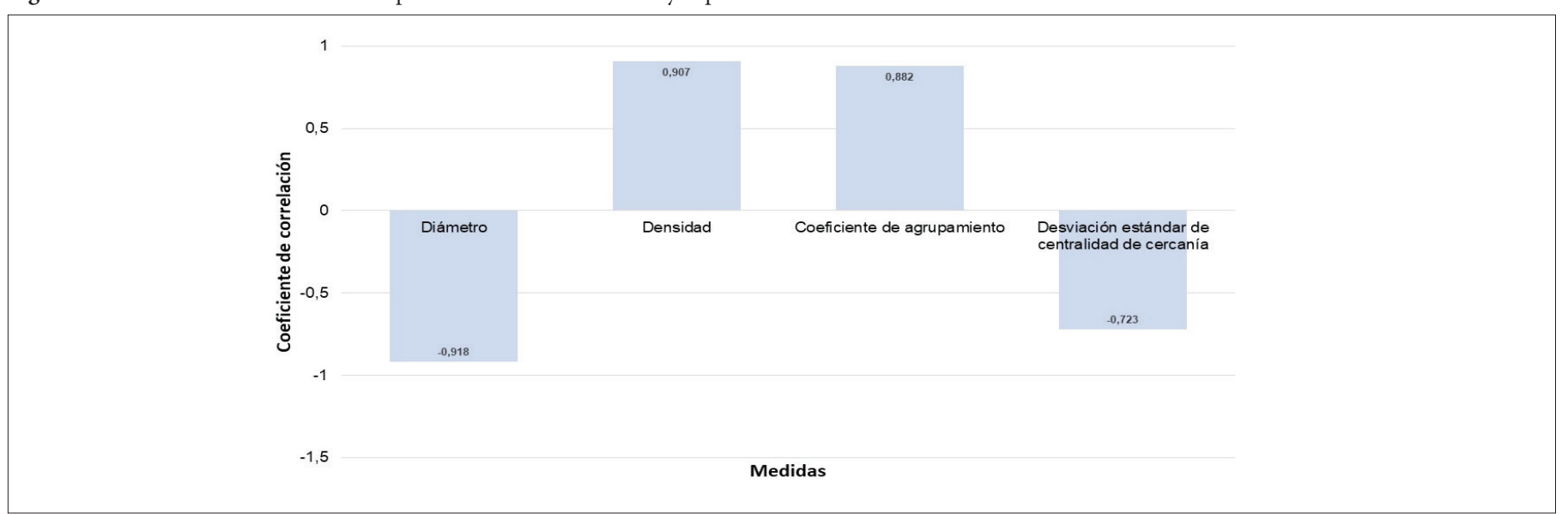

Fuente: elaboración propia

Como lo muestra la Figura 4, dos de las medidas- densidad y coeficiente de agrupamiento- muestran una correlación positiva con la productividad, lo que implica que el aumento de estas variables se asocia con un aumento de la productividad. Las otras dos medidas diámetro y desviación estándar de centralidad de cercanía- muestran una correlación negativa, lo que significa que su aumento se asocia con una disminución de la productividad.

Por otro lado, los resultados del agrupamiento difuso muestran que al agrupar las empresas en tres conjuntos, cuatro organizaciones no alcanzan el umbral mínimo de pertenencia fijado en 0,8 . Sin embargo, con cuatro conjuntos todas las empresas alcanzan este umbral y por esta razón se estableció en cuatro el número de conjuntos requeridos para agrupar a las empresas según productividad. De manera similar, cuando se agrupan las empresas según medidas de red también se observa que existen empresas, en este caso seis, que no muestran clara pertenencia a ningún conjunto cuando hay tres conjuntos. Pero al aumentar a cuatro conjuntos todas ellas las organizaciones se ubican claramente en uno de los conjuntos. Las Tablas 5 y 6 ilustran estos resultados.

Tabla 5: Coeficiente de pertenencia de cada empresa a cada conjunto, con tres y cuatro conjuntos, para agrupamiento según productividad. Las zonas sombreadas muestran las empresas que sin una clara pertenencia a algún conjunto.

\begin{tabular}{|c|c|c|c|c|c|c|}
\hline \multirow[b]{2}{*}{ Empresa } & \multicolumn{3}{|c|}{ Coeficientes de pertenencia con 3 conjuntos } & \multicolumn{3}{|c|}{ Coeficientes de pertenencia con 4 conjuntos } \\
\hline & $\mathrm{C} 1$ & $\mathrm{C} 2$ & $\mathrm{C} 3$ & $\mathrm{C} 1$ & $\mathrm{C} 2$ & $\mathrm{C} 3$ \\
\hline 1 & 0,95 & 0,01 & 0,04 & 0,99 & 0,00 & 0,01 \\
\hline 2 & 0,99 & 0,00 & 0,00 & 0,99 & 0,01 & 0,00 \\
\hline 4 & 1,00 & 0,00 & 0,00 & 0,99 & 0,00 & 0.01 \\
\hline 5 & 0,00 & 1,00 & 0,00 & 0,00 & 0,96 & 0,03 \\
\hline 8 & 0,81 & 0,14 & 0,05 & 0,92 & 0,02 & 0,03 \\
\hline 9 & 0,19 & 0,69 & 0,12 & 0,00 & 0,00 & 1,00 \\
\hline 10 & 0,14 & 0,74 & 0,12 & 0,00 & 0,00 & 0,99 \\
\hline 11 & 0,01 & 0,05 & 0,94 & 0,01 & 0,06 & 0,02 \\
\hline 12 & 0,01 & 0,95 & 0,05 & 0,00 & 1,00 & 0,00 \\
\hline 17 & 0,01 & 0,87 & 0,12 & 0,00 & 0,96 & 0,01 \\
\hline 18 & 0,00 & 0,98 & 0,01 & 0,00 & 1,00 & 0,00 \\
\hline 19 & 0,00 & 0,01 & 0,99 & 0,00 & 0,02 & 0,01 \\
\hline 20 & 0,00 & 0,01 & 0,99 & 0,00 & 0,01 & 0,00 \\
\hline 21 & 0,00 & 0,00 & 1,00 & 0,00 & 0,00 & 0,00 \\
\hline 22 & 0,00 & 0,97 & 0,03 & 0,00 & 1,00 & 0,00 \\
\hline 23 & 0,00 & 0,00 & 1,00 & 0,00 & 0,00 & 0,00 \\
\hline 24 & 0,00 & 0,01 & 0,99 & 0,00 & 0,02 & 0,01 \\
\hline 25 & 0,00 & 0,95 & 0,05 & 0,00 & 1,00 & 0,00 \\
\hline
\end{tabular}

ISSN: 0718-2724. (http://jotmi.org)

Journal of Technology Management \& Innovation ( ) Universidad Alberto Hurtado, Facultad de Economía y Negocios. 


\begin{tabular}{llll}
\hline \multicolumn{5}{c}{ Coeficientes de pertenencia con 3 conjuntos } \\
\hline 26 & 0,00 & 0,00 & 1,00 \\
\hline 27 & 0,15 & 0,72 & 0,13 \\
\hline 28 & 0,14 & 0,68 & 0,18 \\
\hline 29 & 0,01 & 0,05 & 0,94 \\
\hline 30 & 0,01 & 0,95 & 0,05 \\
\hline 31 & 0,00 & 1,00 & 0,00 \\
\hline 32 & 0,00 & 1,00 & 0,00 \\
\hline 33 & 0,00 & 0,99 & 0,01 \\
\hline 34 & 0,00 & 0,00 & 1,00 \\
\hline 35 & 0,00 & 0,01 & 0,99 \\
\hline 36 & 0,00 & 0,01 & 0,99 \\
\hline 37 & 0,00 & 1,00 & 0,00 \\
\hline 38 & 0,00 & 0,99 & 0,01 \\
\hline 39 & 0,00 & 1,00 & 0,00 \\
\hline 40 & 0,00 & 0,99 & 0,01 \\
\hline
\end{tabular}

\begin{tabular}{ccc} 
Coeficientes de pertenencia con $\mathbf{4}$ conjuntos \\
\hline 0,00 & 0,00 & 0,00 \\
\hline 0,00 & 0,00 & 0,99 \\
\hline 0,00 & 0,00 & 0,99 \\
\hline 0,01 & 0,06 & 0,02 \\
\hline 0,00 & 1,00 & 0,00 \\
\hline 0,99 & 0,01 & 0,00 \\
\hline 0,00 & 0,96 & 0,03 \\
\hline 0,00 & 0,96 & 0,03 \\
\hline 0,00 & 0,00 & 0,99 \\
\hline 0,00 & 0,00 & 0,99 \\
\hline 0,00 & 0,00 & 0,99 \\
\hline 0,00 & 1,00 & 0,00 \\
\hline 0,00 & 0,02 & 0,01 \\
\hline 0,00 & 0,01 & 0,00 \\
\hline 0,00 & 0,00 & 0,00 \\
\hline
\end{tabular}

Fuente: elaboración propia.

Tabla 6: Coeficiente de pertenencia de cada empresa a cada conjunto, con tres y cuatro conjuntos, para agrupamiento según medidas de red. Las zonas sombreadas muestran las empresas sin una clara pertenencia a algún conjunto.

\begin{tabular}{|c|c|c|c|c|c|c|}
\hline \multicolumn{4}{|c|}{ Coeficientes de pertenencia con 3 conjuntos } & \multicolumn{3}{|c|}{ Coeficientes de pertenencia con 4 conjuntos } \\
\hline Empresa & $\mathrm{C} 1$ & $\mathrm{C} 2$ & $\mathrm{C} 3$ & $\mathrm{C} 1$ & $\mathrm{C} 2$ & $\mathrm{C} 3$ \\
\hline 1 & 0,96 & 0,01 & 0,03 & 0,98 & 0,00 & 0,01 \\
\hline 2 & 0,96 & 0,01 & 0,02 & 0,99 & 0,00 & 0,01 \\
\hline 3 & 1,00 & 0,00 & 0,00 & 0,94 & 0,00 & 0,04 \\
\hline 4 & 1,00 & 0,00 & 0,00 & 0,94 & 0,00 & 0,05 \\
\hline 5 & 0,02 & 0,91 & 0,08 & 0,01 & 0,9 & 0,03 \\
\hline 6 & 0,00 & 0,99 & 0,01 & 0,00 & 0,99 & 0,00 \\
\hline 7 & 0,01 & 0,94 & 0,05 & 0,01 & 0,91 & 0,01 \\
\hline 8 & 0,96 & 0,01 & 0,03 & 0,98 & 0,00 & 0,01 \\
\hline 9 & 0,45 & 0,11 & 0,44 & 0,01 & 0,00 & 0,97 \\
\hline 10 & 0,71 & 0,07 & 0,22 & 0,03 & 0,00 & 0,95 \\
\hline 11 & 0,03 & 0,21 & 0,77 & 0,02 & 0,03 & 0,05 \\
\hline 12 & 0,00 & 1,00 & 0,00 & 0,00 & 1,00 & 0,00 \\
\hline 13 & 0,02 & 0,08 & 0,91 & 0,01 & 0,04 & 0,02 \\
\hline 14 & 0,00 & 1,00 & 0,00 & 0,00 & 1,00 & 0,00 \\
\hline 15 & 0,00 & 0,99 & 0,01 & 0,00 & 0,99 & 0,00 \\
\hline 16 & 0,02 & 0,08 & 0,90 & 0,00 & 0,07 & 0,03 \\
\hline 17 & 0,00 & 0,99 & 0,01 & 0,00 & 0,99 & 0,00 \\
\hline 18 & 0,00 & 1,00 & 0,00 & 0,00 & 0,99 & 0,00 \\
\hline 19 & 0,01 & 0,03 & 0,96 & 0,01 & 0,04 & 0,04 \\
\hline 20 & 0,01 & 0,03 & 0,96 & 0,02 & 0,04 & 0,04 \\
\hline 21 & 0,02 & 0,08 & 0,91 & 0,01 & 0,04 & 0,02 \\
\hline 22 & 0,02 & 0,78 & 0,20 & 0,01 & 0,92 & 0,04 \\
\hline 23 & 0,01 & 0,03 & 0,96 & 0,01 & 0,03 & 0,04 \\
\hline 24 & 0,01 & 0,03 & 0,96 & 0,02 & 0,04 & 0,01 \\
\hline 25 & 0,00 & 1,00 & 0,00 & 0,00 & 1,00 & 0,00 \\
\hline 26 & 0,01 & 0,03 & 0,96 & 0,01 & 0,03 & 0,04 \\
\hline 27 & 0,28 & 0,29 & 0,43 & 0,01 & 0,00 & 0,97 \\
\hline 28 & 0,57 & 0,16 & 0,27 & 0,03 & 0,00 & 0,96 \\
\hline 29 & 0,02 & 0,24 & 0,74 & 0,04 & 0,01 & 0,04 \\
\hline 30 & 0,00 & 1,00 & 0,00 & 0,00 & 1,00 & 0,00 \\
\hline 31 & 0,00 & 1,00 & 0,00 & 0,94 & 0,00 & 0,04 \\
\hline 32 & 0,00 & 1,00 & 0,00 & 0,00 & 0,99 & 0,00 \\
\hline 33 & 0,00 & 0,99 & 0,01 & 0,00 & 0,99 & 0,00 \\
\hline
\end{tabular}




\begin{tabular}{cccc}
\hline \multicolumn{5}{c}{ Coeficientes de pertenencia con 3 conjuntos } \\
\hline 34 & 0,00 & 0,00 & 1,00 \\
\hline 35 & 0,00 & 0,01 & 0,99 \\
\hline 36 & 0,00 & 0,01 & 0,99 \\
\hline 37 & 0,00 & 1,00 & 0,00 \\
\hline 38 & 0,00 & 0,99 & 0,01 \\
\hline 39 & 0,00 & 1,00 & 0,00 \\
\hline 40 & 0,00 & 0,99 & 0,01 \\
\hline
\end{tabular}

Fuente: elaboración propia.

En la Tabla 7 se presentan los valores de los centroides de los conjuntos resultantes. Como se señaló previamente en la metodología, los centroides se configuran como los valores representativos de cada conjunto.
Coeficientes de pertenencia con 4 conjuntos

\begin{tabular}{lll}
0,01 & 0,00 & 0,97 \\
\hline 0,03 & 0,00 & 0,96 \\
\hline 0,00 & 0,00 & 0,99 \\
\hline 0,00 & 1,00 & 0,00 \\
\hline 0,00 & 0,02 & 0,01 \\
\hline 0,00 & 0,01 & 0,00 \\
\hline 0,00 & 0,00 & 0,00 \\
\hline
\end{tabular}

Cada uno de los cuatro conjuntos se asocia con un nivel de productividad: medio, medio bajo, medio alto y alto, y con un valor estándar para cada una de las medidas de red empleadas como indicadores de la EC.

Tabla 7: Valores de los centroides para cada una de las cuatro categorías de empresas obtenidas a través del agrupamiento difuso.

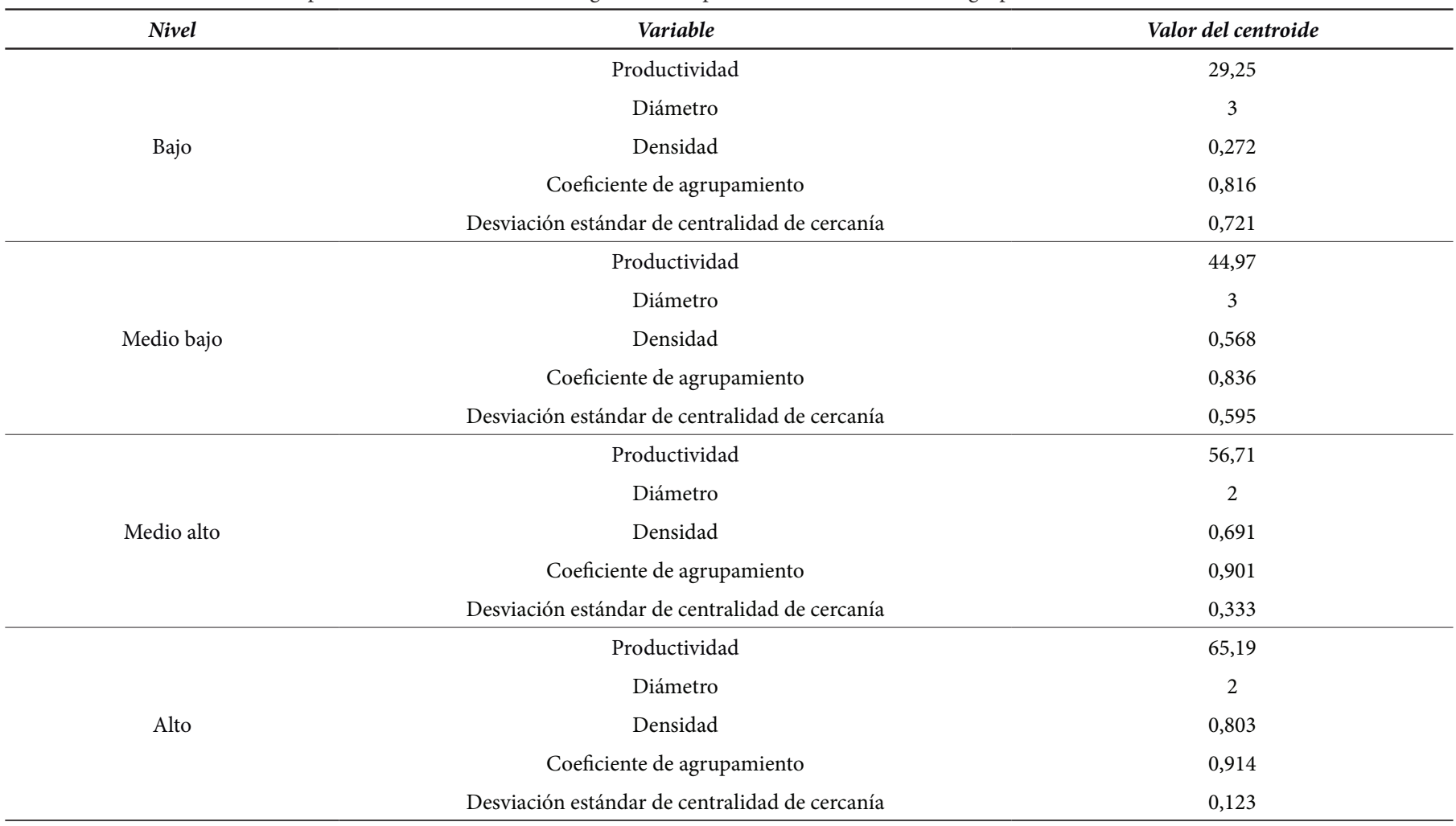

Fuente: elaboración propia.

Finalmente, al evaluar la coincidencia entre conjuntos creados según productividad y según medidas de red, se encuentra que con tres conjuntos la coincidencia es de 0,55 , y con cuatro conjuntos la coincidencia es de 1. Estos datos señalan que cuando se al tener cuatro conjuntos es posible obtener una coincidencia perfecta entre los agrupamientos según productividad y los agrupamientos según medidas de red.

\section{Discusiones}

Los resultados del test de Shapiro Wilk, presentados en la Tabla 4 demuestran que las variables no tienen distribución normal. La distribución no normal es consistente con la complejidad de la realidad de las empresas y su GC, señalando que existen diversas fuerzas intervinientes en la productividad. Sus dinámicas y efectos no se pueden explicar por procesos puramente aleatorios.

Por otro lado, los resultados muestran que las medidas evaluadas satisfacen los dos criterios requeridos y presentados previamente en la sección de Metodología: correlación con la productividad y capacidad para discriminar por nivel de productividad. Por lo tanto, se considera que las medidas son adecuadas para conformar el modelo. El criterio de correlación se demuestra con la alta y significativa correlación obtenida entre cada medida y la productividad, lo que significa que los resultados obtenidos y mostrados en la Figura 4 no son producto del 
azar. Tales resultados también indican que variaciones de las medidas se corresponden con variaciones de la productividad. Consecuentemente, la variación de una medida puede asociarse con el incremento o disminución de la productividad, de acuerdo con el sentido de la correlación (positiva o negativa). El sentido positivo de la correlación en la densidad y el coeficiente de agrupamiento implica que la productividad tiende a aumentar cuando aumentan la cantidad y cercanía de las relaciones entre los trabajadores en los procesos de EC. Mientras que el sentido negativo de la correlación en el diámetro y la desviación estándar de la centralidad de cercanía implican que la productividad tiende a disminuir mientras más difícil sea para los trabajadores acceder a otros procesos de EC en los que no participaron.

Los mencionados resultados son coherentes con la literatura sobre facilitadores de la transformación del conocimiento. La densidad y el coeficiente de agrupamiento, aspectos relacionados con la amplia interacción entre los trabajadores, aumentan cuando aumenta la productividad evidenciando la necesidad de fomentar estos fenómenos. En contraste, el diámetro y la desviación estándar de centralidad de cercanía aumentan cuando disminuyen la productividad. Dado que estas medidas se relacionan con menor posibilidad de acceder a los procesos de EC creados por otros trabajadores, se considera que estas medidas evalúan aspectos que deben desincentivarse.

Por otro lado, las Tablas 5 y 6 muestran que con cuatro conjuntos todas las empresas presentaron un coeficiente de agrupamiento superior al umbral de 0,8. Esta clara pertenencia a alguno de los conjuntos indica que cuatro conjuntos es una cantidad adecuada para clasificar a las empresas scon base en productividad y medidas de red. Con cuatro conjuntos también se obtiene una coincidencia perfecta, lo que añade evidencia a la pertinencia de emplear cuatro conjuntos para clasificar a las empresas. Lo que implica que cada uno de los grupos posee niveles similares de productividad, así como características similares en sus procesos de EC.

Los anteriores resultados indican que se requiere más de una medida de red para representar adecuadamente la complejidad de los procesos de EC. El modelo planteado aprovecha cuatro medidas diferentes, asociadas con diferentes facilitadores de la transformación del conocimiento y responsables de evaluar diferentes dimensiones del proceso. Es por ello que el incremento de la productividad debe interpretarse como correlacionada con un conjunto de características, de manera colectiva, y no como rasgos aislados que tienen por sí solos una correlación con la productividad.

Por último, los resultados señalan que la forma como las medidas de red aumentan o disminuyen también es relevante para el modelo. Si todas las medidas tuvieran un comportamiento constante (de aumento o disminución) con cada nuevo vínculo, podría esperarse una tendencia similar en la productividad: aumentar o disminuir con cada nuevo vínculo-proceso de EC. Pero como se describió en la sección de metodología, mientras para dos medidas cada vínculo implica una variación constante, para las otras dos medidas la variación se determina por la posición del nuevo vínculo. En la realidad de la empresa, esto podría interpretarse como la relevancia relativa de un proceso de EC de acuerdo con los cargos involucrados. Así, un documento creado por los cargos $A$ y $B$ puede aportar más que un documento creado por $B$ y $C$. En consecuencia, los resultados sugieren que para una empresa podría resultar más valioso y requerir menos recursos determinar qué trabajadores deberían trabajar juntos en un proceso de EC, y fomentar esas interacciones, en lugar de promover masivamente que todos los trabajadores interactúen entre sí.

\section{Conclusiones}

Este trabajo planteó como objetivo diseñar un modelo de red para evaluar la relación entre la EC y la productividad en pymes. Los hallazgos permiten concluir que es posible aprovechar la ciencia de redes para representar y evaluar de forma cuantitativa la complejidad de los procesos de EC y su relación en la productividad. De esta forma, este trabajo contribuye a superar los principales retos de implementar este proceso en las empresas. Por un lado, aporta evidencia sobre la relación fuerte y significativa que existe la EC y la productividad. Pero también se muestra que las empresas con determinadas características de la EC pueden tener mejor productividad, y otras con características opuestas pueden tener el nivel de desempeño más bajo.

Las capacidades del modelo pueden seguir siendo evaluadas con una muestra de mayor tamaño, incluyendo organizaciones de diferente tipo: otros tamaños (microempresas, grandes empresas), otras regiones, y sectores como público o civil. En este trabajo tampoco se consideró el número de documentos que generan juntos cada par de trabajadores, interactúan, o el hecho de que existan algunos documentos más relevantes que otros. Estos rasgos de la EC que pueden evaluarse asignando peso a los vínculos no fueron tenidos en cuenta en este estudio. En futuras investigaciones podrían incluirse para evaluar su impacto sobre la productividad. Finalmente, puede ampliarse el alcance de la investigación a un nivel explicativo para determinar si, además de correlación, y bajo determinadas circunstancias existe una relación causal entre las características de la EC y la productividad. En concordancia con esto, futuras investigaciones pueden orientarse a la evaluación del modelo enriqueciendo la muestra, caracterizar los conjuntos de empresas según su estructura de transformación y evaluar el nivel de influencia de la EC sobre la productividad.

\section{Referencias}

Alkhazali, Z., Aldabbagh, I., \& Abu-Rumman, A. (2019). Tqm Potential Moderating Role to the Relationship between Hrm Practices, Km Strategies and Organizational Performance: The Case of Jordanian Banks. Academy of Strategic Management Journal, 18(3), 1-16.

Alvarenga Neto, R. \& Choo, C. W. (2011). Expanding the concept of Ba: managing enabling contexts in knowledge organizations. Perspectivas em Ciência da Informação, 16(3), 2-25.

Barabási, A. L. (2016). Network science. Cambridge university press.

Bashir, M., \& Farooq, R. (2019). The synergetic effect of knowledge management and business model innovation on firm competence. International Journal of Innovation Science. 
Bezdek, J. C., Ehrlich, R., \& Full, W. (1984). FCM: The fuzzy c-means clustering algorithm. Computers \& Geosciences, 10(2-3), 191-203.

Boccaletti, S., Latora, V., Moreno, Y., Chavez, M., \& Hwang, D. U. (2006). Complex networks: Structure and dynamics. Physics reports, 424(4-5), 175-308.

Broekel, T., \& Mueller, W. (2018). Critical links in knowledge networks-What about proximities and gatekeeper organisations?. Industry and Innovation, 25(10), 919-939.

Casaburi, G., Crespi, G., De León, I. L., Fernández, J., Garone, L. F., Grazzi, M., ... \&

Chatterjee, A., Pereira, A., \& Sarkar, B. (2018). Learning transfer system inventory (LTSI) and knowledge creation in organizations. The Learning Organization.

Dahou, K., Hacini, I., \& Burgoyne, J. (2019). Knowledge management as a critical success factor in developing international companies' organizational learning capability. Journal of Workplace Learning.

Deni, A., Priansa, D. J., Darmo, I. S., Saribanon, E., Riswanto, A., Sumaryadi, S., \& Ramdan, A. M. (2020). Organizational Competency and Innovation Capability: The Influence of Knowledge Management on Business Performance. Quality-Access to Success, 21(179).

Dini, M., Stumpo, G., \& Italiana, I. C. (2011). Políticas para la innovación en las pequeñas y medianas empresas en América Latina.

Ferreira, J., Mueller, J. and Papa, A. (2018), "Strategic knowledge management: theory, practice and future challenges", Journal of Knowledge Management, Vol. 24 No. 2, pp. 121-126. https://doi. org/10.1108/JKM-07-2018-0461

Frohmann, A., Mulder, N., \& Olmos, X. (2018). Promoción de la innovación exportadora: instrumentos de apoyo a las pymes.

Hahn, M. H., Lee, K. C., \& Lee, D. S. (2015). Network structure, organizational learning culture, and employee creativity in system integration companies: The mediating effects of exploitation and exploration. Computers in Human Behavior, 42, 167-175.

Jiang, S., Zhang, X., Cheng, Y., Xu, D., De Pablos, P. O., \& Wang, X. (2019). Dynamic impact of social network on knowledge contribution loafing in mobile collaboration: a hidden Markov model. Journal of Knowledge Management.

Kao, S. C., \& Wu, C. (2016). The role of creation mode and social networking mode in knowledge creation performance: Mediation effect of creation process. Information \& Management, 53(6), 803-816.

Kyakulumbye, S., Pather, S., \& Jantjies, M. (2019). Knowledge Creation in a Participatory Design Context: The use of Empathetic Participatory Design. Electronic Journal of Knowledge Management, 17(1).
Nonaka, I., \& Takeuchi, H. (1995). The knowledge-creating company: how Japanese companies create the dynamics of innovation. Oxford: Oxford University Press.

Nonaka, I., Toyama, R., \& Nagata, A. (2000). A firm as a knowledgecreating entity: a new perspective on the theory of the firm. Industrial and corporate change, 9(1), 1-20.

Nonaka, I., Von Krogh, G., \& Voelpel, S. (2006). Organizational knowledge creation theory: Evolutionary paths and future advances. Organization studies, 27(8), 1179-1208.

Obeidat, A. (2019). IT Adaption with Knowledge Conversion Process (SECI)?. Management Science Letters, 9(13), 2241-2252.

Phelps, C., Heidl, R., \& Wadhwa, A. (2012). Knowledge, networks, and knowledge networks: A review and research agenda. Journal of management, 38(4), 1115-1166.

Rai, R., \& Prakash, A. (2012). A relational perspective to knowledge creation: Role of servant leadership. Journal of Leadership Studies, 6(2), 61-85.

Shin, S. K., \& Kook, W. (2014). Can knowledge be more accessible in a virtual network?:Collective dynamics of knowledge transfer in a virtual knowledge organization network. Decision Support Systems, 59, 180-189.

Smith, E. A. (2001). The role of tacit and explicit knowledge in the workplace. Journal of knowledge Management. 5(4), pp. 311-321

Sutanto, J., Tan, C. H., Battistini, B., \& Phang, C. W. (2011). Emergent leadership in virtual collaboration settings: A social network analysis approach. Long Range Planning, 44(5-6), 421-439.

Takeuchi, H. (2001). Towards a Universal Management Concept of Knowledge. Managing industrial knowledge: creation, transfer and utilization. In Nonaka, I. and Teece, D. (Ed) Management industrial knowledge. Creation, transfer and utilization. Sage Publications.

Uwasomba, C. F., Seeam, P., Bellekens, X., \& Seeam, A. (2016, August). Managing knowledge flows in Mauritian multinational corporations: Empirical analysis using the SECI model. In 2016 IEEE International Conference on Emerging Technologies and Innovative Business Practices for the Transformation of Societies (EmergiTech) (pp. 341-344). IEEE.

Yun, S., \& Lee, J. (2013). An innovation network analysis of science clusters in South Korea and Taiwan. Asian Journal of Technology Innovation, 21(2), 277-289. 
